\title{
Salud y enfermedad en nuestras poblaciones originarias: ¿qué los hace ser más susceptibles o resistentes a ciertas enfermedades prevalentes?
}

\author{
Juan Francisco Miquel Poblete
}

Departamento de Gastroenterología.

Facultad de Medicina, Pontificia Universidad Católica de Chile

En la presente edición de la Revista Chilena de Cardiología se publican dos interesantes artículos relacionados con la salud de dos poblaciones indígenas contemporáneas en dos extremos de nuestro país, la Atacameña en la II Región y la Pehuenche en la VIII Región. A. Zapata y col. comparan el estado nutricional entre Atacameños rurales y urbanos adultos (promedio de edad similar, 58 a 59 años), de aparente similar grado de ancestría indígena. La definición de Atacameño se basó en la presencia de apellidos de origen Atacameño en los sujetos de estudio y sus antecesores (padres, abuelos). La prevalencia de sobrepeso y obesidad evaluada por IMC $(\mathrm{kg} / \mathrm{mt} 2)$ fue muy elevada y similar en ambos grupos estudiados (70\% y 75\% en rurales y urbanos, respectivamente). La obesidad abdominal o centrípeta, medida por circunferencia de cintura, fue significativamente mayor en los Atacameños rurales comparados con los urbanos. A su vez, el colesterol plasmático total fue significativamente mayor en los Atacameños urbanos comparado con los rurales. Estos datos si bien interesantes por provenir de grupos indígenas específicos rurales y urbanos, no difieren significativamente de la información generada en la Segunda Encuesta Nacional de Salud 20092010 (www.minsal.cl), donde se comunican prevalencias de sobrepeso-obesidad para estos grupos etarios en la población general adulta Chilena, similares a las descritas por Zapata y col. Si bien Zapata y col. no especifican cómo se midió la circunferencia de cadera (central o a la altura de las caderas, ver Segunda Encuesta Nacional de Salud), la obesidad abdominal en los Atacameños rurales pareciera ser mayor a lo reportado para la población general de esta región.

En el segundo artículo, C. Navarrete y col. comparan la prevalencia de hipertensión arterial (HTA) entre individuos Pehuenche mayores de 15 años con lo que denominan individuos mestizos y occidentales que viven en las mismas localidades rurales del alto Bío-Bio. El tamaño muestral fue predefinido en forma adecuada, y la categorización Pehuenche, Mestizo y Occidental se basó en la existencia o no de apellidos indígenas según CONADI. El mensaje más importante de este trabajo es que la prevalencia de HTA fue similar en los 3 grupos étnicos y no difiere significa-

\section{Correspondencia:}

Profesor titular

Depto. De Gastroenterología, Escuela de Medicina

Pontificia Universidad Católica de Chile

jfmiquel@med.puc.cl 
tivamente de la reportada para la población general Chilena en estos rangos etario (Segunda Encuesta Nacional de Salud 2009-2010).

Ambos trabajos apuntan a evaluar condiciones de salud/enfermedad en grupos indígenas chilenos y fueron efectuados en el contexto de Medicina Comunitaria o Familiar, lo que de por sí es muy meritorio. Es de interés para nuestro país conocer en detalle las patologías de ocurrencia frecuente (enfermedades prevalentes) e infrecuente (enfermedades raras, generalmente monogénicas) que afectan con especial fuerza a nuestras poblaciones originarias. Si bien esta información epidemiológica tiene consecuencias prácticas mediatas que permiten, por ejemplo, proponer el desarrollo de programas de atenciones especiales en salud a grupos étnicos minoritarios afectados con mayor fuerza por ciertas patologías específicas, la información generada de estudios comparativos de prevalencia de enfermedades entre diferentes grupos étnicos tiene proyecciones mucho más vastas.

En las últimas décadas ha existido un interés creciente en el estudio de fenotipos o enfermedades que afectan con especial intensidad a grupos indígenas de América $^{1}$ y que los diferencian de poblaciones Caucásicas $^{2}$. A su vez, existe información creciente de la transmisión de alguno de estos fenotipos o enfermedades, a las poblaciones mestizas contemporáneas con ancestro indígena americano que componen la mayor parte de las poblaciones de Centro y Sudamérica ${ }^{3-6}$. Estudio recientes han confirmado que América fue poblada por una primera migración de poblaciones provenientes de Asia hace aproximadamente $15.000 \mathrm{a}$ 20.000 años ADP, llamados "primeros Americanos"7 Esta primera migración dio origen a la mayor parte de las poblaciones nativas de América del Norte, Centro y Sur, incluido nuestra parte del continente. Dos migraciones posteriores habrían dado origen a algunas poblaciones originarias restringidas al extremo Norte de América, en Canadá y la Antártica. Esta información de carácter antropológico y origen poblacional nos permite entender el porqué las poblaciones indígenas contemporáneas de América que descienden de estos "primeros Americanos" y las poblaciones Mestizas contemporáneas con ancestro indígena, pudieran compartir una peculiar susceptibilidad a desarrollar algunos fenotipos y enfermedades prevalentes, la mayoría de ellas relacionadas a ciertas condiciones metabólicas comunes ${ }^{8-11}$. Nuestro Departamento de Gas- troenterología ha contribuido a generar conocimiento y hemos seguido de cerca los estudios que señalan la especial susceptibilidad de nuestra población indígena e hispana (Mestiza) a desarrollar enfermedades de la vesícula biliar, en particular la colelitiasis y sus consecuencias clínicas incluido el elevado riesgo a desarrollar cáncer de la vesícula biliar. Hemos demostrado que la colelitiasis afecta a $\sim 30 \%$ de nuestra población adulta, existiendo una clara mayor susceptibilidad en población chilena Mapuche comparada con la población general hispana o mestiza ${ }^{12}$. En particular, tanto mujeres como hombres Mapuches desarrollan esta enfermedad a edades más tempranas de la vida. Es destacable, que esta elevada prevalencia de colelitiasis es compartida entre Indígenas de Norte y Sud América, y existe desde la época precolombina, siendo por lo tanto éste un fenotipo que probablemente fue introducido en nuestro continente por los "primeros americanos" y ha persistido hasta la actualidad ${ }^{13}$. La heredabilidad de esta enfermedad ha sido estudiada formalmente en poblaciones Mestizas (mexicanos americanos), pero no en poblaciones indígenas ${ }^{14,15}$. Estos estudios proponen que el 40 a $60 \%$ de la prevalencia de colelitiasis es explicada por factores genéticos o heredables, lo que contrasta con sólo un $25 \%$ en población Europea ${ }^{16}$. Nosotros postulamos que estos genes que denominamos "litogénicos", son privativos de las poblaciones indígenas americanas, y han sido transferidas a poblaciones mestizas con ancestro Amerindio ${ }^{12,13}$.

Es interesante destacar que los principales factores de riesgo de la colelitiasis se relacionan con el exceso de peso y la resistencia insulínica $(\mathrm{RI})^{17,18}$. Diferentes estudios que se iniciaron en los años 70 destacan que las poblaciones indígenas de América y sus descendientes Mestizos, presentan una mayor tendencia a desarrollar exceso de peso, y más específicamente, a desarrollar obesidad centrípeta o abdominal (denominada también adiposidad visceral ${ }^{19}$. Existen abundantes evidencias en la literatura que señalan que la obesidad abdominal es uno de los principales factores de riesgo para desarrollar RI y eventualmente diabetes mellitus tipo 2 (DM2) ${ }^{20,21}$. Como es de esperar, la obesidad abdominal es también un factor de riesgo a desarrollar colelitiasis, especialmente en poblaciones con ancestro Amerindio ${ }^{19,22-24}$. En este escenario, el estudio de A. Zapata y col., muestra resultados en consonancia con lo reportado, señalando que los Atacameños tienen obesidad abdominal. Lamentablemente, no es 
claro en este estudio, si el grupo de estudio rural (con mayor obesidad abdominal) es comparable al urbano en términos de etnicidad, dado que no se utilizaron marcadores más objetivos de grado de ancestría Amerindia, como grupos sanguíneos ${ }^{12}$ o marcadores genéticos más sofisticados ${ }^{25,26}$. El estudio de C. Navarrete y col., no evaluó marcadores más objetivos de etnicidad en los tres grupos estudiados y lamentablemente no entrega detalles y parámetros antropométricos en los diferentes grupos étnicos estudiados, dado que el objetivo fue el estudio de prevalencia de HTA.

Existen evidencias bien documentadas que las poblaciones Amerindias tienen un perfil de colesterol plasmático que difiere de poblaciones Caucásicas o Mestizas, con menores niveles de colesterol-total, colesterol-LDL y mayores niveles de colesterol-HDL ${ }^{12}$, 27,28 . Este perfil metabólico es consistente con un menor riesgo cardiovascular, y sus mecanismos no ha sido aclarados, pudiendo ser genéticos y/o ambientales. Por otro lado, un elegante trabajo publicado recientemente por C.A. Celis-Morales y col $^{29}$, estudia en mayor profundidad el riesgo de desarrollar RI en Indígenas chilenos (Mapuches) comparado con chilenos de origen europeo. Específicamente comparan a subgrupos rurales y urbanos de estas etnias y evalúan la interacción del factor étnico y ambiental en el riesgo a presentar RI, medido por el índice de resistencia insulínica HOMA (HOMAIR). Lo destacable de este trabajo, es que se confirma la significativa mayor susceptibilidad de la población Mapuche a desarrollar RI al compararla con una etnia europea chilena. A su vez, los autores demuestran por primera vez una significativa interacción etnicidad $\mathrm{x}$ ambiente, siendo los Mapuches especialmente susceptibles a desarrollar RI cuando modifican su ambiente de rural a urbano. Por último, este estudio muestra una significativa influencia de la etnicidad sobre el efecto de la adiposidad y actividad física sobre los valores de HOMAIR, con un mayor efecto en Mapuches comparados con Europeos. Es destacable además, que en este estudio se confirma que los chilenos de etnia Mapuche no difieren de la Europea en cifras de presión arterial, lo que es consistente con el trabajo reportado aquí de $\mathrm{C}$. Navarrete y col.. Por otro lado, C.A. Celis-Morales y col. entregan información adicional que evidencia que la etnia Mapuche presenta un significativo mayor grado de sobrepeso y adiposidad comparado con el grupo chileno europeo, lo que es consistente con lo reportado por nosotros ${ }^{12}$.

Un estudio también reciente demuestra que la etnia Mapuche es un factor de riesgo independiente para el desarrollo de Síndrome Metabólico ${ }^{30}$. Como es sabido, bajo el concepto de Síndrome Metabólico se incluye las variables metabólicas previamente analizadas, como perímetro de cintura, presión arterial, niveles de lípidos plasmáticos y glicemia ${ }^{31,32}$. Existen diferentes definiciones consensuadas por paneles de expertos internacionales, pero todas están destinadas a identificar sujetos en mayor riesgo a desarrollar DM2 y enfermedades cardiovasculares. En suma, las evidencias disponibles indican que nuestras poblaciones originarias tienen una mayor susceptibilidad a desarrollar obesidad abdominal y RI, pero tienen un perfil lipídico más favorable (protector de riesgo CV) y no presentarían una mayor susceptibilidad a desarrollar HTA. En el balance general de estas condiciones metabólicas, el pertenecer a la etnia Mapuche se asociaría a un mayor riesgo de desarrollar síndrome metabólico. Sin embargo, las evidencias disponibles señalan que nuestras poblaciones originarias no presentan una especial mayor susceptibilidad a desarrollar DM2 ${ }^{12}$ y no existe suficiente información sobre la prevalencia/incidencia de enfermedades CV y mortalidad por eventos $\mathrm{CV}$ en nuestras poblaciones originarias, comparada con la población Mestiza o de origen europeo chilena. Carecemos también de información local que compare el riesgo a desarrollar eventos $\mathrm{CV}$ en nuestra población mayoritaria que es Mestiza de origen biparental (Mapuche-Español) con población Chilena de ancestro mayoritariamente Europeo. Es necesario desarrollar estudios tendientes a responder estas preguntas, dado que independiente de la existencia o no de factores de riesgo a enfermedades $\mathrm{CV}$ y mortalidad CV que han sido definidos para poblaciones Caucásicas, es posible imaginar que las poblaciones indígenas de América y Mestizas tengan una susceptibilidad que difiere de otras poblaciones a desarrollar los eventos $\mathrm{CV}$ finales (infarto al miocardio, accidentes vasculares encefálicos, etc).

Otro grupo de enfermedades prevalentes que parecen tener un perfil diferente entre poblaciones nativas Americanas y Mestizas o hispanas con ancestro Amerindio al compararlas con etnias caucásicas o negra, son algunas enfermedades hepáticas crónicas ${ }^{33,34}$. Ha sido bien documentado en los últimos años, que las poblaciones hispánicas con ancestro Amerindio, tienen una mayor 
susceptibilidad a desarrollar hígado graso no alcohólico (NAFL) y estetatohepatitis (NASH). A su vez, la población hispana tienen una mayor prevalencia de daño hepático por alcohol y una mayor tendencia a desarrollar fibrosis frente a una infección por virus $\mathrm{C}$ de hepatitis y mayor riesgo de desarrollar carcinoma hepatocelular. Es interesante destacar que tanto la presencia de NAFL/NASH y colelitiasis confieren globalmente mayor riesgo a desarrollar enfermedades cardiovasculares y mortalidad por enfermedades cardiovasculares ${ }^{35-38}$. De hecho, ambas enfermedades han sido consideradas como parte del síndrome metabólico. Así, dado que nuestras poblaciones indígenas e hispana con ancestro Amerindio tienen mayor riesgo a desarrollar colelitiasis, NAFL/NASH, obesidad abdominal y RI, es probable que sean más susceptibles a sufrir eventos $\mathrm{CV}$ y mortalidad de origen $\mathrm{CV}$, lo que debe ser evaluado en trabajos prospectivos y sistemáticos futuros.

\section{Referencias:}

1. NEEL JV. Health and disease in unacculturated Amerindian populations. Ciba Found Symp 1977:155-68.

2. CHAKRABORTY R, FERRELL RE, STERN MP, HAFFNER SM,HAZUDAHP, ROSENTHAL M. Relationship of prevalence of non-insulin-dependent diabetes mellitus to Amerindian admixture in the Mexican Americans of San Antonio, Texas. Genet Epidemiol 1986;3:435-54.

3. ALARCON GS, BEASLEY TM, ROSEMAN JM, MCGWIN G, JR., FESSLER BJ, BASTIAN HM, et al. Ethnic disparities in health and disease: the need to account for ancestral admixture when estimating the genetic contribution to both (LUMINA XXVI). Lupus 2005;14:867-8.

4. MOLOKHIA M, MCKEIGUE P. Risk for rheumatic disease in relation to ethnicity and admixture. Arthritis Res 2000;2:115-25.

5. TSENG M, WILLIAMS RC, MAURER KR, SCHANFIELD MS, KNOWLER WC, EVERHART JE. Genetic admixture and gallbladder disease in Mexican Americans. Am J Phys Anthropol 1998;106:361-71.

6. HANIS CL, CHAKRABORTY R, FERRELL RE, SCHULL

WJ. Individual admixture estimates: disease associations and individual risk of diabetes and gallbladder disease among Mexican-Americans in Starr County, Texas. Am J Phys Anthropol 1986;70:433-41.

7. REICH D, PATTERSON N, CAMPBELL D, TANDON A, MAZIERES S, RAY N, et al. Reconstructing Native American population history. (2012) Nature advance online publication: doi: 10.1038 /nature11258

8. DENNY CH, HOLTZMAN D, GOINS RT, CROFT JB. Disparities in chronic disease risk factors and health status between American Indian/Alaska Native and White elders: findings from a telephone survey, 2001 and 2002. Am J Public Health 2005;95:825-7.

9. JARVIS JN, CLELAND SY. Rheumatic disease in Native Ame- rican children: opportunities and challenge. Curr Rheumatol Rep 2003;5:471-6

10. SEWELL JL, MALASKY BR, GEDNEY CL, GERBER TM, BRODY EA, PACHECO EA, et al. The increasing incidence of coronary artery disease and cardiovascular risk factors among a Southwest Native American tribe: the White Mountain Apache Heart Study. Arch Intern Med 2002;162:1368-72.

11. REEVES MJ, REMINGTON PL, NASHOLD R, PETE J Chronic disease mortality among Wisconsin Native American Indians, 1984-1993. Wis Med J 1997;96:27-32.

12. MIQUEL JF, COVARRUBIAS C, VILLAROEL L, MINGRONE G, GRECO AV, PUGLIELLI L, et al. Genetic epidemiology of cholesterol cholelithiasis among Chilean Hispanics, Amerindians, and Maoris. Gastroenterology 1998;115:937-46.

13. NERVI F, MIQUEL JF, MARSHALL G. The Amerindian epidemics of cholesterol gallstones: the North and South connection. Hepatology 2003;37:947-8.

14. DUGGIRALA R, MITCHELL BD, BLANGERO J, STERN MP. Genetic determinants of variation in gallbladder disease in the Mexican-American population. Genet Epidemiol 1999;16:191-204.

15. PUPPALA S, DODD GD, FOWLER S, ARYA R, SCHNEIDER J, FAROOK VS, et al. A genomewide search finds major susceptibility Loci for gallbladder disease on chromosome 1 in mexican americans. Am J Hum Genet 2006;78:377-92.

16. LAMMERT F, SAUERBRUCH T. Mechanisms of disease: the genetic epidemiology of gallbladder stones. Nat Clin Pract Gastroenterol Hepatol 2005;2:423-33.

17. AMIGO L, ZANLUNGO S, MENDOZA H, MIQUEL JF, NERVI F. Risk factors and pathogenesis of cholesterol gallstones: state of the art. Eur Rev Med Pharmacol Sci 1999;3:241-6.

18. MAURER KR, EVERHART JE, KNOWLER WC, SHAWKER TH, ROTH HP. Risk factors for gallstone disease in the Hispanic populations of the United States. Am J Epidemiol 1990;131:836-44.

19. DIEHL AK, STERN MP. Special health problems of Mexican- 
Americans: obesity, gallbladder disease, diabetes mellitus, and cardiovascular disease. Adv Intern Med 1989;34:73-96.

20. GRUNDY SM. Metabolic complications of obesity. Endocrine 2000;13:155-65.

21. DESPRES JP. Intra-abdominal obesity: an untreated risk factor for Type 2 diabetes and cardiovascular disease. J Endocrinol Invest 2006;29:77-82.

22. TSAI CJ, LEITZMANN MF, WILLETT WC, GIOVANNUCCI EL. Prospective study of abdominal adiposity and gallstone disease in US men. Am J Clin Nutr 2004;80:38-44.

23. CAREY MC, PAIGEN B. Epidemiology of the American Indians' burden and its likely genetic origins. Hepatology 2002;36:781-91.

24. HAFFNER SM, DIEHL AK, STERN MP, HAZUDA HP. Central adiposity and gallbladder disease in Mexican Americans. Am J Epidemiol 1989;129:587-95.

25. MCKEIGUE PM. Mapping genes that underlie ethnic differences in disease risk: methods for detecting linkage in admixed populations, by conditioning on parental admixture. Am J Hum Genet 1998;63:241-51.

26. MITCHELL BD, WILLIAMS-BLANGERO S, CHAKRABORTY R, VALDEZ R, HAZUDA HP, HAFFNER SM, et al.A comparison of three methods for assessing Amerindian admixture in Mexican Americans. Ethn Dis 1993;3:22-31.

27. STOCKINS B, LARENAS G, CHARLES M, STANDEN D, ESPINOZA O, ILLESCA M, et al. [Serum lipids and blood pressure levels in the +Mapuche population living in the Chilean region of Araucania]. Rev Med Chil 1998;126:1291-9.

28. KRAWCZYK M, LUTJOHANN D, SCHIRIN-SOKHAN R, VILLARROEL L, NERVI F, PIMENTEL F, et al. Phytosterol and cholesterol precursor levels indicate increased cholesterol excretion and biosynthesis in gallstone disease. Hepatology 2012;55:1507-17.

29. CELIS-MORALES CA, PEREZ-BRAVO F, IBANES L, SAN-
ZANAR, HORMAZABALE, ULLOAN, et al. Insulin resistance in Chileans of European and indigenous descent: evidence for an ethnicity x environment interaction. PLoS One;6:e24690.

30. PHILCO LP, SERON SP, MUÑOZ NS, NAVIA BP, LANAS ZF. [Risk factors for metabolic syndrome in a case control study in Temuco, Chile]. Rev Med Chil;140:334-9.

31. ALBERTI KG, ZIMMET P, SHAW J. Metabolic syndrome--a new world-wide definition. A Consensus Statement from the International Diabetes Federation. Diabet Med 2006;23:469-80.

32. [A worldwide consensus definition for the metabolic syndrome]. Rev Panam Salud Publica 2005;18:451-4.

33. CARRION AF, GHANTA R, CARRASQUILLO O, MARTIN P. Chronic liver disease in the Hispanic population of the United States. Clin Gastroenterol Hepatol;9:834-41; quiz e109-10.

34. PEREZ A, ANZALDUA M, MCCORMICK J, FISHERHOCH S. High frequency of chronic end-stage liver disease and hepatocellular carcinoma in a Hispanic population. J Gastroenterol Hepatol 2004;19:289-95.

35. MCKIMMIE RL, DANIEL KR, CARR JJ, BOWDEN DW, FREEDMAN BI, REGISTER TC, et al. Hepatic steatosis and subclinical cardiovascular disease in a cohort enriched for type 2 diabetes: the Diabetes Heart Study. Am J Gastroenterol 2008;103:3029-35.

36. GOESSLING W,MASSARO JM, VASANRS, D'AGOSTINO RB, SR., ELLISON RC, FOX CS. Aminotransferase levels and 20-year risk of metabolic syndrome, diabetes, and cardiovascular disease. Gastroenterology 2008;135:1935-44, 1944 e1.

37. TARGHER G, ARCARO G. Non-alcoholic fatty liver disease and increased risk of cardiovascular disease. Atherosclerosis 2007;191:235-40

38. VILLANOVA N, MOSCATIELLO S, RAMILLI S, BUGIANESI E, MAGALOTTI D, VANNI E, et al. Endothelial dysfunction and cardiovascular risk profile in nonalcoholic fatty liver disease. Hepatology 2005;42:473-80. 


\title{
Artículo de Revisión
}

\section{¿Cuál es el riesgo cardiovascular del adulto asintomático?}

\author{
Tomás Romero.

\section{Introducción:}

Esta pregunta, que desde sus comienzos (1947, 1948) fue central en los estudios de los Siete Países y Framingham ${ }^{1,2}$, implica, además de un gran interés epidemiológico, la búsqueda de una respuesta a un problema no resuelto en la prevención cardiovascular: La enfermedad coronaria determina aproximadamente el $80 \%$ de los casos de muerte súbita en adultos y en la mayoría de ellos es su primera y última manifestación clínica ${ }^{3 "}$..

Los "factores de riesgo" cardiovascular, identificados progresivamente a partir de esos estudios y que permitieron desarrollar el concepto de que los cambios en el estilo de vida son primordiales en el control del riesgo cardiovascular, se han organizado en tablas predictivas de un evento coronario intentando no sólo responder a esa interrogante, sino promover estrategias terapéuticas de prevención primaria y secundaria.
Clasificación del Riesgo: Aportes y Limitaciones.

Las estimaciones originales del riesgo cardiovascular en el estudio Framingham (una cohorte en sus comienzos casi exclusivamente blanca y de origen anglo-sajón) han sido adaptadas con posterioridad en diferentes regiones del mundo, a grupos raciales-étnicos diferentes y en relación al género, con la introducción de variantes reflejando esas diferencias pero esencialmente usando los mismos conceptos o agregando otros factores de riesgo (Sociedad Europea de Cardiología- SCORE, REGICOR, ACRS, score de Reynolds, entre otras) $)^{4,5,6,7,8,9}$
El riesgo de sufrir un evento coronario al cabo de 10 años basado en las escalas Framingham y revisadas el año 2004 por el Tercer Panel de Tratamiento de Adultos (ATP-III), fue clasificado en cuatro categorías: riesgo bajo, intermedio, intermedio alto y alto $(<5 \%$, $6-10 \%, 11-20 \%,>20 \%$, respectivamente). Aunque estos niveles de riesgo son estimaciones aproximadas, con un grado de precisión predictiva no mayor de $75 \%$, el seguimiento de la cohorte Framingham por más de 60 años, y posteriormente de otras, ha validado su uso generalizado en la práctica médica ${ }^{5,7,8,9.10}$. La gran virtud del uso de las Tablas Framingham, y de sus modifi-

Correspondencia:

Tomás Romero.

Clinical Profesor School of Medicine

University of California

San Diego USA

Email: tromero560@aol.com 